\title{
The Sub-Parsec Structure of Accretion Disks as Revealed by VLBI Imaging of Free-Free Absorption
}

\author{
D.L. Jones, A.E. Wehrle, B.G. Piner, \& D.L. Meier \\ Jet Propulsion Laboratory, California Institute of Technology, \\ 4800 Oak Grove Drive, Pasadena, CA 91109, USA
}

\begin{abstract}
The physical conditions in the inner parsec of accretion disks believed to orbit the central black holes in active galactic nuclei can be probed by imaging the absorption of background radio emission by ionized gas in the disk. High angular resolution radio observations of several nearby galaxies at multiple frequencies have revealed evidence for freefree absorption by disks or tori of ionized gas. The depth and angular width of the absorption increases with decreasing frequency. The longest possible baselines are needed to provide adequate angular resolution at low frequencies where the effects of free-free absorption are most evident. Recent results from VSOP as well as ground-based VLBI observations of the nearby galaxy NGC 4261 illustrate the critical importance of high angular resolution at frequencies below $10 \mathrm{GHz}$.
\end{abstract}

\section{Introduction}

Evidence for free-free absorption of radio emission from parsec-scale jets has been found in a number of galactic nuclei, including one of the strongest compact radio sources (3C84; Walker et al. 1999) and the closest active galaxy (Centaurus A; Jones et al. 1996). In all clear cases a parsec-scale counterjet is detectable, and it is the emission from the counterjet that appears to be absorbed by intervening ionized gas. The fact that the counterjet but not the approaching jet shows absorption suggests that the absorbing material exists in the equatorial plane of the central black hole - that is, in an accration disk or torus.

The relatively nearby $(40 \mathrm{Mpc})$ elliptical galaxy NGC 4261 is known from HST observations to contain a central black hole with a mass of $5 \times 10^{8} \mathrm{M}_{\odot}$ and a nearly edge-on nuclear disk of gas and dust on a scale of $\sim 100 \mathrm{pc}$. This galaxy is associated with the low luminosity (FR-I) radio source $3 \mathrm{C} 270$, whose large-scale morphology suggests that the radio axis is close to the plane of the sky. Consequently NGC 4261 is a good candidate for the detection of free-free absorption by ionized gas in an inner accretion disk. At a distance of $40 \mathrm{Mpc}$, 1 milliarcsecond (mas) corresponds to $0.2 \mathrm{pc}$.

Previous VLBA observations of NGC 4261 at $8.4 \mathrm{GHz}$ revealed a pc-scale radio jet and counterjet aligned with the kpc-scale jet, and a narrow gap in radio brightness at the base of the pc-scale counterjet. We identified this gap as the signature of free-free absorption by a nearly edge-on inner disk. 


\section{Observations}

We observed NGC 4261 at 1.6 and $4.9 \mathrm{GHz}$ on 22 and 27 June 1999 respectively with HALCA and a large ground array. At $1.6 \mathrm{GHz}$ the ground array included 7 VLBA antennas plus Shanghai, Kashima, and the DSN 70-m Tidbinbilla antennas, and at $4.9 \mathrm{GHz}$ we used $8 \mathrm{VLBA}$ antennas plus the phased VLA. Data were recorded as two $16-\mathrm{MHz}$ bandwidth channels with 2-bit sampling by the Mark-III/VLBA systems. In addition, we observed with the VLBA only at 8.4 $\mathrm{GHz}$ on 16 October 1999 as part of a multi-epoch monitoring program (Piner, et al. 2000). This experiment recorded $64 \mathrm{MHz}$ total bandwidth. Data from all three frequencies were correlated at the VLBA processor in Socorro.

Fringe-fitting was carried out in AIPS after applying a priori amplitude calibration. Fringes were found to all antennas at $1.6 \mathrm{GHz}$ except HALCA, but the signal/noise ratio to Shanghai and Kashima was very low and these data were not used. The a priori amplitude calibration for Tidbinbilla was dramatically incorrect for unknown reasons. We calibrated Tidbinbilla by imaging the compact structure of the source using VLBA data, then holding the VLBA antenna gains fixed and allowing the Tidbinbilla gain to vary. This produced a good match in correlated flux density where the projected VLBA and Tidbinbilla baselines overlap. At $4.9 \mathrm{GHz}$ fringes were found to all antennas, including HALCA. A similar correction to the a priori amplitude calibration for HALCA and the phased VLA was applied. At $8.4 \mathrm{GHz}$ no correction to the a priori amplitude calibration was required. Left circular polarization was used for all three experiments.

Difmap was used for detailed data editing, self-calibration, and image deconvolution. Imaging within Difmap used uniform weighting with the weight of HALCA data increased by a factor of 500 . Several iterations of phase-only self calibration, followed by amplitude self calibration iterations with decreasing time scales, resulted in good fits $\left(\chi^{2} \approx 1\right)$ between the source models and the data.

\section{Results}

\section{1. $\quad$ 1.6 GHz}

Although our image at $1.6 \mathrm{GHz}$ does not include data from HALCA, it does have more than twice the angular resolution of our previous $1.6 \mathrm{GHz}$ image (Jones and Wehrle 1997) due to the addition of Tidbinbilla. Our previous, lower resolution, image showed a symmetric structure, with the jet and counterjet extending west and east from the core. No evidence for absorption is seen in this image. However, with higher resolution we do detect a narrow gap in emission just east of the core, at the base of the counterjet (see figure 1). The width of the gap is less than 2 mas.

\section{2. $4.9 \mathrm{GHz}$}

We detected fringes to HALCA at $4.9 \mathrm{GHz}$ when the projected Earth-space baselines were less than one Earth diameter. The HALCA data fills in the $(u, v)$ 


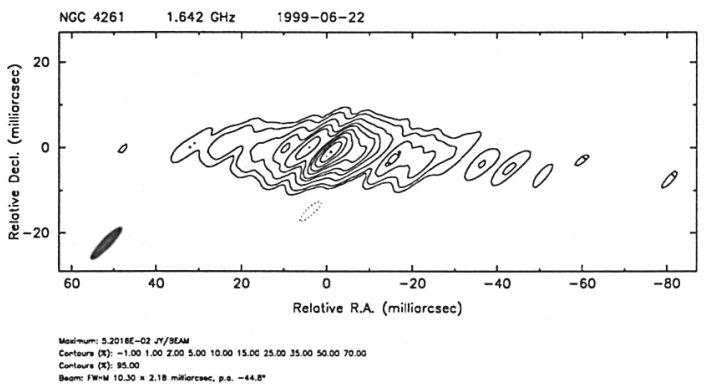

Figure 1. VLBA+Tidbinailla image of NGC 4261 at $1.6 \mathrm{GHz}$. The contours are $-1,1,2,5,10,15,25,35,50,70$, and $95 \%$ of the peak (52 $\mathrm{mJy} /$ beam), and the beam is $10.3 \times 2.2 \mathrm{mas}$ in $\mathrm{PA}=-45^{\circ}$.

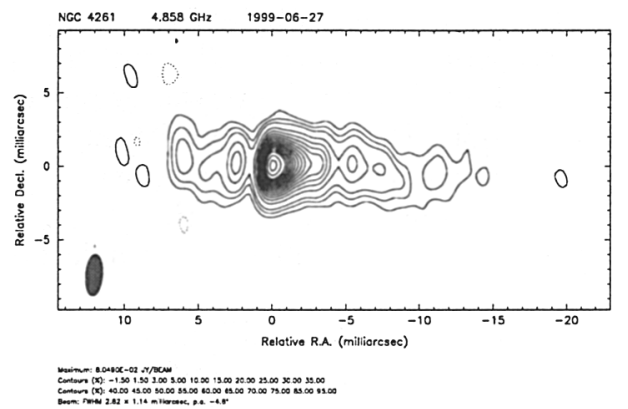

Figure 2. VLBA+VLA+HALCA image of NGC 4261 at $4.9 \mathrm{GHz}$. The contours are $-1.5,1.5,3,5,10,15,25,35,50,70$, and $95 \%$ of the peak $(80 \mathrm{mJy} /$ beam $)$, and the beam is $2.82 \times 1.14$ mas in $\mathrm{PA}=-2.5^{\circ}$.

coverage hole between continental VLBA baselines and those to Mauna Kea, and also increases the north-south resolution by a factor of two. Our $4.9 \mathrm{GHz}$ image is shown in Figure 2. Note that the gap in emission is again seen just east of the peak.

\section{3. $8.4 \mathrm{GHz}$}

The $8.4 \mathrm{GHz}$ image shown in figure 3 is the most recent of three VLBA images we have obtaind at this frequency over a 4 year period. The angular resolution at 4.9 and $8.4 \mathrm{GHz}$ is similar, and the observing epochs are close enough in time to avoid significant changes in source structure (Piner, et al. 2000). A careful comparison of brightness along the radio axis at 4.9 and $8.4 \mathrm{GHz}$ shows that the gap is both deeper and wider at $4.9 \mathrm{GHz}$, as expected from free-free absorption. The region of the gap has a very inverted spectrum, the brightest peak (core) has a slightly less inverted spectrum, and the distant parts of both the jet and counterjet have steep spectra. 


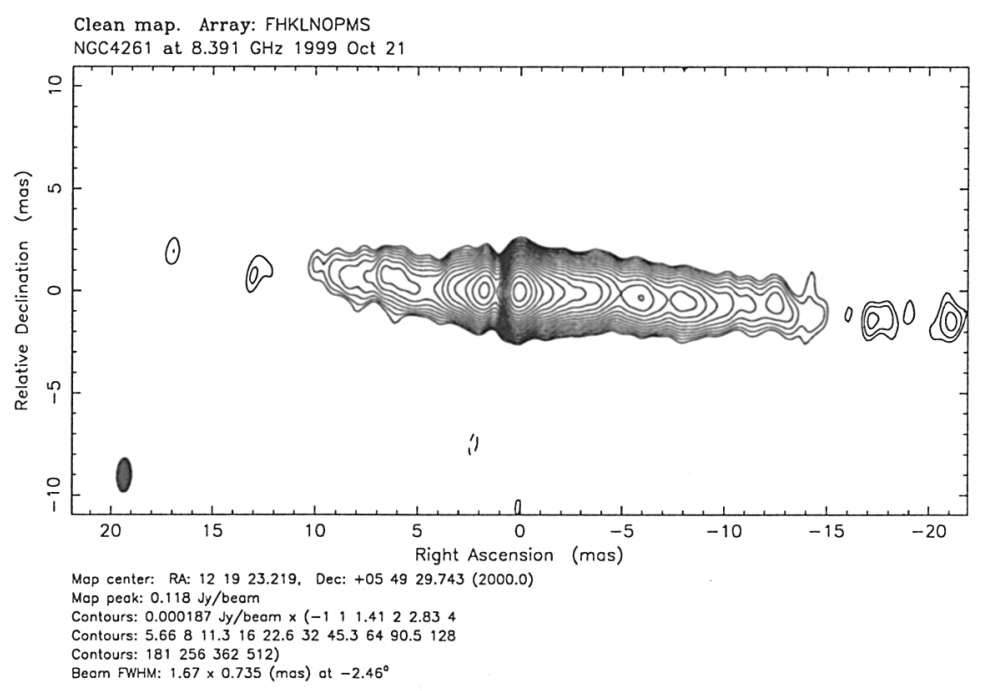

Figure 3. VLBA image of NGC 4261 at $8.4 \mathrm{GHz}$. The contours increase in steps of $\sqrt{2}$ starting at $\pm 1 \%$ of the peak $(118 \mathrm{mJy} /$ beam $)$, and the beam is $1.67 \times 0.74$ mas in $\mathrm{PA}=-2.5^{\circ}$.

\section{Summary}

Our observations at $1.6,4.9$, and $8.4 \mathrm{GHz}$ appear to confirm the free-free absorption explanation for the sub-parsec radio morphology in NGC 4261. Measurements of the optical depth in the absorbed region and the distance between the absorption and the core as a function of frequency will allow the radial distribution of electron density in the inner parsec of the disk to be determined.

Acknowledgments. We gratefully acknowledge the VSOP Project, which is led by the Japanese Institute of Space and Astronautical Science in cooperation with many organizations and radio telescopes around the world. The VLBA is a facility of the National Radio Astronomy Observatory, which is operated by Associated Universities, Inc., under a cooperative agreement with the U.S. National Science Foundation. This research was carried out at the Jet Propulsion Laboratory, California Institute of Technology, under contract with the U.S. National Aeronautics and Space Administration.

\section{References}

Jones, D.L. \& Wehrle, A.E. 1997, ApJ, 484, 186

Jones, D.L., Wehrle, A.E., Meier, D.L., \& Piner, B.G. 2000, ApJ, 534, 165

Piner, B.G., Jones, D.L., \& Wehrle, A.E. 2000, in preparation 